\title{
Diseño y evaluación de un programa para la mejora de la condición física en Educación Primaria: efecto sobre la autoestima y la intención de ser activo
}

\section{Design and evaluation of a program for the improvement of the physical fitness in Primary Education}

\author{
David Zamorano García*, Juan Gregorio Fernández Bustos y Juan Pablo Vállez Gómez
}

Facultad de Educación de Albacete. Universidad de Castilla - La Mancha (España).

\begin{abstract}
Resumen: El objetivo fue diseñar un programa para mejorar la condición física y evaluar su efecto sobre la intencionalidad de seguir siendo activo y autoestima general. Se diseńó un programa de 8 semanas de actividades para mejorar la condición física en Educación Física, además de actividad física deportiva en los recreos. El diseño cuasiexperimental incluyó 70 escolares (39 grupo experimental; 31 grupo control) de 6 o de Educación Primaria. Como instrumentos de medida se utilizaron la batería ALPHA-Fitness de alta prioridad, la Escala de intencionalidad de ser físicamente activo en Educación Primaria, y el Cuestionario Multimedia y Multilingüe de Evaluación de la Autoestima. Se encontró efectividad del programa en todas las variables estudiadas, en especial en la mejora de la capacidad cardiorrespiratoria, la capacidad de salto y la dimensión socioafectiva de la autoestima. Se destaca la importancia de estos programas en la mejora de la salud física, psicológica y social del alumnado.

Palabras clave: condición física, autoestima, salud, educación primaria.
\end{abstract}

Abstract: The objective was to design a program to improve physical fitness and evaluate its effect on the intent to remain active and general self-esteem. An 8-week program of activities was designed to improve physical fitness in Physical Education, in addition to sports physical activity at recess. The quasi-experimental design included 70 schoolchildren (39 experimental group; 31 control group) from 6th grade of Primary Education. The high priority ALPHA-Fitness battery, the intentionality scale of being physically active in Primary Education, and the Multimedia and Multilingual Selfesteem Evaluation Questionnaire were used as measuring instruments. The effectiveness of the program was found in all the variables studied, especially in the improvement of cardiorespiratory capacity, jumping capacity and the socio-affective dimension of self-esteem. The importance of these programs in the improvement of the physical, psychological and social health of the students is highlighted.

Key words: physical fitness, self-esteem, health, primary school.

\section{Introducción}

La actividad física (AF) se asocia positivamente con indicadores del estado de salud a nivel físico, psicológico y social. Por ejemplo, existen evidencias que vinculan la $\mathrm{AF}$ con múltiples beneficios fisiológicos (Janssen y LeBlanc, 2010) como la composición corporal (Bicanin, Milenkovic, Radovanovic, Gajevic y Ivanovic, 2018), el aumento de la capacidad aeróbica (Mocha, Garcés, Medina, Castro y Suntasig, 2018) o una mejor condición física (CF) (Guillamón, García-Cantó, Pérez y Rodríguez, 2015). En el plano psicológico son conocidos sus efectos positivos sobre indicadores de ansiedad y depresión (Strong et al., 2005; Janssen y Leblanc, 2010), mejoras en función cognitiva y rendimiento académico (Diamond, 2015; Álvarez-Bueno et al., 2017), o sobre el propio autoconcepto (Fernández-Bustos, Infantes-Paniagua, Cuevas y Contreras, 2019).

Estas evidencias han alentado a la OMS (2010) a proponer recomendaciones en cuanto a cantidad y tipo de AF, especialmente en escolares, existiendo, sin embargo, evidencias de su incumplimiento (Currie, Gabhainn y Godeau, 2008). Además, la AF realizada en las clases de Educación Física (EF) no

Dirección para correspondencia [Correspondence address]: David Zamorano García. Universidad de Castilla - La Mancha (España) E-mail: david.zamorano@uclm.es contribuye decisivamente a la consecución de las citadas recomendaciones, bien por el escaso aprovechamiento de práctica de AF real en clases de EF, o por el escaso tiempo disponible dentro del currículo escolar (Camacho-Miñano, FernándezGarcía, Ramírez y Blández, 2013).

Diversos han sido los programas implementados desde la EF escolar con resultados positivos en torno a aumentar la calidad (UNESCO, 2015) y cantidad de la AF (Lonsdale et al., 2013). Muchos de ellos se han centrado en el trabajo de mejora de la CF orientada a la salud (CFS), pues ésta se considera un indicador objetivo del estado de salud de los niños (Janz, Dawson y Mahoney, 2002) y uno de los más importantes marcadores de salud (Ortega, Ruiz, Castillo y Sjöström, 2008).

Además de AF, la EF debe promover la práctica más allá de sus clases, creando el ambiente y motivación apropiados (Faison-Hodge y Porretta, 2004) para aumentar la intención de sus alumnos de seguir siendo activo fuera de la escuela y aumentar su autoestima (Fernández-Bustos et al., 2019). Estudios previos han encontrado relación entre una mejor $\mathrm{CF}$, autoconcepto e intención de seguir siendo activo (Guillamón, García-Cantó y Carrillo, 2019; Kyle, Hernández, Reigal y Morales., 2016). 
Partiendo de estas premisas, el presente trabajo tiene como objetivos diseñar e implementar un programa de AF en horario escolar, incluyendo iniciativas tanto durante las clases de EF como durante los periodos de recreo del alumnado, así como valorar sus efectos sobre la mejora de la CF, la intencionalidad de seguir siendo físicamente activo y la autoestima.

\section{Método}

El diseño de este estudio es cuasiexperimental con pruebas pretest y postest en una muestra no probabilística a conveniencia, incluyendo grupos control y experimental.

\subsection{Participantes}

Participaron en la investigación 70 alumnos de $6^{\circ}$ de Educación Primaria de entre 11 y 13 años $(M=11,45$ ańos $)$ de dos centros de Educación Primaria de Albacete (España). 39 de ellos pertenecían al grupo experimental (24 hombres, $15 \mathrm{mu}$ jeres) y 31 al grupo control (14 hombres, 17 mujeres).

\subsection{Instrumentos}

Evaluación de la CF: se empleó la batería ALPHA-Fitness de alta prioridad (Ruiz et al., 2011) que valora los siguientes componentes de la CFS: composición corporal (índice de masa corporal y perímetro de cintura), capacidad aeróbica (test de ida y vuelta de 20 metros) y capacidad músculo-esquelética (fuerza de prensión manual y salto de longitud con pies juntos).

Evaluación de la intencionalidad de mantenerse físicamente activo: se valoró por medio de la Escala de Intencionalidad para ser Físicamente Activo en Educación Primaria, adaptada y traducida al español por Estero, Oliva y Lucas (2013) partiendo de la Intention to be Physically Active Scale (Hein, Müür, y Koka, 2004). Está compuesta de cinco ítems para medir la intención de la persona de ser físicamente activo, precedidos de la frase "Respecto a tu intención de practicar deporte...", con respuestas tipo Likert de 5 opciones (1=totalmente en desacuerdo; 5 =totalmente de acuerdo).

Evaluación de la autoestima: se utilizó el Cuestionario Multimedia y Multilingüe de Evaluación de la Autoestima (CMMEA) (Ramos, 2008). Este cuestionario está basado en el modelo multidimensional del autoconcepto propuesto originalmente por Shavelson, Hubner y Stanton (1976) y toma en consideración los factores socioafectivos (17 ítems, p. ej. 6. "Soy feliz"), académico (9 ítems, p. ej. 2. "Estoy contento/a con mis notas") y físico-deportivo (4 ítems, p. ej. 10. "Soy torpe para la mayoría de los deportes"). Este cuestionario se compone de 30 preguntas en total y con respuesta tipo Likert con 3 opciones (siempre-a veces-nunca).

Evaluación de la AF: del Physical Activity Questionna- rie for Children (PAQ-C) adaptado y validado en población español por Manchola-González, Bagur-Calafat y GirabentFarrés (2017), se administró el ítem 6 (En los últimos 7 días, ¿cuántas tardes hiciste deporte, baile o jugaste a juegos en los que estuviste muy activo?). Éste ítem se responde en una escala del 1 al 5, aumentando el número en función de la frecuencia con que realizaron AF.

\subsection{Procedimiento}

Se informó a los equipos directivos y maestros de EF de los centros participantes sobre los objetivos y características de la investigación. Todos ellos aceptaron la participación voluntaria. Previamente a la recogida de datos, participantes y padres fueron informados de objetivos, procedimientos, riesgos y beneficios de la investigación. Los participantes fueron voluntarios entre aquellos que proporcionaron el consentimiento informado. La investigación se llevó a cabo bajo las normas deontológicas recogidas por la Declaración de Helsinki (revisión Hong-Kong, 1989), las recomendaciones de Buena Práctica Clínica de la CEE (documento 111/3976/88 de julio de 1990), y la normativa legal española que regula la investigación clínica en humanos (Real Decreto 561/1993 sobre ensayos clínicos), garantizándose durante todo el proceso anonimato y confidencialidad de los datos.

Se llevó a cabo una toma de datos pretest y postest de tres sesiones cada una. Antes de comenzar la intervención se aplicó la batería ALPHA-Fitness de alta prioridad a partir del protocolo propuesto por Ortega et al. (2011). En la primera sesión se midió la composición corporal y, en la segunda, la capacidad músculo-esquelética y capacidad aeróbica. En la tercera se cumplimentaron los cuestionarios CMMEA, MIFA y PAQ-C...

El programa de intervención tuvo una duración de 8 semanas a razón de 2 sesiones semanales en clase de EF, más una actividad complementaria semanal durante los recreos. En las clases de EF se trabajó principalmente la resistencia aeróbica, dedicando una actividad en cada sesión para la mejora de la fuerza, alternando core, miembro superior y miembro inferior. Además, durante estas clases se utilizaron estrategias metodológicas orientadas a maximizar el tiempo de compromiso motor y de AF de moderada a vigorosa (Abad y Cañada, 2014; Powell, Woodfield y Nevill, 2016), estrategias motivacionales para concienciar sobre la importancia de mantenerse activo físicamente y los beneficios de una buena CFS para la salud presente y futura. Por otra parte, durante los recreos se organizó la práctica de un deporte alternativo (Colpbol) que fuera novedoso, motivante, participativo y adaptable (Fernández-Bustos y Abellán, 2016) basado en el Modelo de Educación Deportiva (Siedentop, 1994). 


\subsection{Análisis de datos}

Se empleó el programa estadístico SPSS versión 24.0. Se llevaron a cabo pruebas de normalidad (Kolmogorov-Smirnov) y homocedasticidad (Leneve). Al no cumplirse estos supuestos se aplicaron pruebas no paramétricas. Se utilizó la prueba U de Mann-Whitney para muestras independientes para los análisis intergrupo (control vs experimental) según las diferentes variables a estudio. Seguidamente, con el fin de comprobar los efectos del programa sobre los participantes del grupo experimental y contrastarlos con el grupo control, se realizó la prueba de Wilcoxon $(\mathrm{Z})$ para muestras relacionadas (pretest vs. postest).

\section{Resultados}

La tabla 1 muestra un muy elevado porcentaje de práctica de actividades físicas muy activas en el grupo experimental antes del inicio del programa. En este grupo, algo más de un $77 \%$ de los participantes eran activos más de 4 veces a la semana, frente al poco más de $48 \%$ del grupo control.

Tabla 1. Frecuencia de actividades en las que se estuvo muy activo en la última semana.

\begin{tabular}{lcccccc}
\hline & Ninguno & $\mathbf{1}$ vez & $\mathbf{2 - 3}$ veces & $\mathbf{4 - 5}$ veces & $\mathbf{6 - 7}$ veces & Total \\
\hline Control & 1 & 2 & 13 & 11 & 4 & 31 \\
$\%$ & 3,23 & 6,45 & 41,94 & 35,48 & 12,9 & 100 \\
Experimental & 1 & 1 & 7 & 16 & 14 & 39 \\
$\%$ & 2,56 & 2,56 & 17,95 & 41,03 & 35,9 & 100 \\
\hline
\end{tabular}

En la tabla 2 se muestran los valores intragrugo (experimental y control) obtenidos antes y después de la intervención, para cada uno de los componentes de la CFS, de la intención de seguir siendo activo y la autoestima. En los resultados obtenidos en la prueba de Wilcoxon para muestras relacionadas existen mejoras en el grupo experimental en relación a las variables sal- to $(\mathrm{Z}=-4,537, \mathrm{p}<.001)$, test $20 \mathrm{~m}(\mathrm{Z}=-4,333, \mathrm{p}<.001)$ y factor socioemocional de la autoestima $(Z=-2,023 c, p<.05)$. En el grupo control no se hallaron diferencias pretest-postest en CF e intención de seguir siendo activo, sin embargo, hubo pérdidas significativas en la autoestima socioafectiva $(Z=-2,582 d$, $\mathrm{p}<.05)$ y académica $(\mathrm{Z}=-2,714 \mathrm{~d}, \mathrm{p}<.01)$.

Tabla 2. Valores intragrupo antes y después de la intervención.

\begin{tabular}{lcccccccccccc}
\hline \multicolumn{1}{c}{ Experimental } & \multicolumn{1}{c}{$M$} & \multicolumn{7}{c}{ Control } \\
\hline & $M$ & DS & $\begin{array}{c}M \\
(\text { Prest })\end{array}$ & DS & $Z$ & $p$ & $\begin{array}{c}M \\
(\text { Pre })\end{array}$ & DS & $\begin{array}{c}M \\
(\text { Post })\end{array}$ & DS & Z & $p$ \\
\hline IMC & 19,46 & 3,01 & 19,40 & 2,94 &, 00 & 1,000 & 19,85 & 3,11 & 19,87 & 3,11 & $-1,38$ &, 166 \\
PC & 67,09 & 7,35 & 67,44 & 6,60 &,- 89 &, 370 & 73,39 & 7,86 & 73,39 & 7,86 &, 00 & 1,000 \\
SPJ & 162,10 & 21,55 & 169,72 & 24,46 & $-4,53$ &, $000^{* * *}$ & 133,90 & 21,15 & 134,13 & 20,48 &,- 80 &, 424 \\
PM & 22,49 & 4,23 & 22,02 & 4,38 & $-1,62$ &, 105 & 21,05 & 4,24 & 21,10 & 4,19 &,- 92 &, 358 \\
20m & 3,50 & 1,89 & 4,42 & 1,96 & $-4,33$ &, $000^{* * *}$ & 3,53 & 1,66 & 3,57 & 1,62 &,- 53 &, 593 \\
ifa1 & 4,49 &, 68 & 4,37 &, 88 &,- 42 &, 669 & 4,48 &, 93 & 4,68 &, 54 & $-1,19$ &, 233 \\
ifa2 & 4,56 &, 85 & 4,47 &, 86 &,- 50 &, 611 & 4,71 &, 74 & 4,61 &, 67 &,- 56 &, 571 \\
ifa3 & 4,26 & 1,14 & 4,26 & 1,08 &,- 13 &, 893 & 4,35 & 1,05 & 4,23 & 1,12 &,- 86 &, 386 \\
ifa4 & 4,31 &, 92 & 4,55 &, 60 & $-1,64$ &, 101 & 4,48 &, 81 & 4,26 &, 89 & $-1,07$ &, 282 \\
ifa5 & 4,05 & 1,10 & 4,29 &, 87 & $-1,64$ &, 101 & 4,10 &, 91 & 4,36 &, 91 &,- 94 &, 344 \\
ifaT & 21,67 & 3,47 & 21,95 & 2,90 &,- 85 &, 392 & 22,13 & 3,44 & 22,13 & 2,93 &,- 18 &, 851 \\
FSoc & 2,70 &, 22 & 2,74 &, 24 & $-2,02$ &, $043 *$ & 2,75 &, 18 & 2,71 &, 19 & $-2,58$ &, $010^{*}$ \\
FAca & 2,54 &, 25 & 2,55 &, 25 &,- 40 &, 687 & 2,55 &, 31 & 2,51 &, 32 & $-2,71$ &, $007^{* *}$ \\
FDep & 2,65 &, 47 & 2,71 &, 44 & $-1,49$ &, 136 & 2,64 &, 44 & 2,61 &, 40 & $-1,00$ &, 317 \\
\hline
\end{tabular}

${ }^{*} p<.05 ;{ }^{* *} p<.01 ;{ }^{* * *} p<.001$. IMC: Índice de Masa Corporal (kg/m²); PC: Perímetro de la cintura (cm); SPJ: Salto a pies juntos (cm); PM: Fuerza de prensión manual (kg); $20 \mathrm{~m}:$ Test de ida y vuelta de 20 metros (palieres completados). ifa 1, 2, 3, 4 y 5: items del 1 al 5 de la escala de intencionalidad de mantenerse físicamente activo; ifa T: puntuación total de la escala de intencionalidad de mantenerse físicamente activo.

FSoc: Factor socioemocional; FAca: Factor académico; FDep: Factor deportivo. 
En la tabla 3, se aprecian los valores obtenidos a partir de la prueba U de Mann-Whitney, que sugieren que en las medias de los datos obtenidos antes de la implementación del programa hay diferencias significativas intergrupo en las variables perímetro cintura $(\mathrm{Z}=-3,113, \mathrm{p}<.01)$ y salto $(\mathrm{Z}=$ $-4,642, \mathrm{p}<.001)$ a favor del grupo experimental. Igualmente, tras la intervención se aumentaron las diferencias intergrupos en el perímetro de la cintura $(Z=-3,201, \mathrm{p}<.001)$ y salto $(Z$ $=-5,262, \mathrm{p}<.001)$, mientras que se advirtieron diferencias en el test de $20 \mathrm{~m}(\mathrm{Z}=-1,998, \mathrm{p}<.05)$.

Tabla 3. Valores intergrupo antes y después de la intervención.

\begin{tabular}{|c|c|c|c|c|}
\hline & \multicolumn{2}{|c|}{ Pre } & \multicolumn{2}{|c|}{ Post } \\
\hline & $Z$ & $P$ & $Z$ & $p$ \\
\hline IMC &,- 857 & ,391 &,- 845 & ,398 \\
\hline PC & $-3,113$ &, $002^{* *}$ & $-3,201$ &, $001^{* * *}$ \\
\hline SPJ & $-4,642$ &, $000^{* * *}$ & $-5,262$ &, $000^{* * *}$ \\
\hline PM & $-1,307$ & 191 & -704 & ,482 \\
\hline $20 \mathrm{~m}$ &,- 297 & ,766 & $-1,998$ &, $046^{*}$ \\
\hline ifa1 &,- 538 &, 590 & $-1,507$ &, 132 \\
\hline ifa2 &,- 916 &, 360 &,- 536 & ,592 \\
\hline ifa3 &,- 303 & ,762 &,- 076 & ,940 \\
\hline ifa4 &,- 954 &, 340 & $-1,235$ &, 217 \\
\hline ifa5 &,- 119 & ,905 &,- 478 & ,633 \\
\hline ifaT &,- 731 & ,465 &,- 435 &, 663 \\
\hline FSoc &,- 667 &, 505 &,- 879 &, 379 \\
\hline FAca &,- 533 & ,594 &,- 179 & ,858 \\
\hline FDep &,- 425 & ,671 & $-1,617$ &, 106 \\
\hline \multicolumn{5}{|c|}{$\begin{array}{l}\text { 20m: Test de ida y vuelta de } 20 \text { metros (palieres completados). ifa 1, 2, 3, } 4 \text { y 5: items del } \\
1 \text { al } 5 \text { de la escala de intencionalidad de mantenerse físicamente activo; ifaT: puntuación } \\
\text { total de la escala de intencionalidad de mantenerse físicamente activo. }\end{array}$} \\
\hline FSoc: Fact & nal; FAca & or académico & ep: Factor & \\
\hline
\end{tabular}

\section{Discusión/conclusión}

El objetivo principal de esta investigación fue diseñar un programa de AF incluyendo iniciativas tanto durante las clases de EF como durante los periodos de recreo del alumnado. Una vez implementado se pretendió valorar su efectividad sobre variables como la mejora de la CF, la intención de seguir siendo activo y la autoestima.

El programa, basado en el trabajo de la CF y optimización del tiempo de compromiso motor en las clases de EF, y el uso recreativo-competitivo en el recreo, mostró mejorar la capacidad cardiorrespiratoria (test de ida y vuelta $20 \mathrm{~m}$ ), la fuerza de salto y el componente socioafectivo de la autoestima. Los resultados hallados van en línea con los obtenidos en otros estudios semejantes donde los programas fueron efectivos en la mejora de la resistencia (Eather, Morgan y Revalds, 2016; Mocha et al., 2018) y la capacidad de salto (Eather et al., 2016).

El programa no pareció tener ningún efecto sobre la mejora de la fuerza manual, como también confirmaron estudios previos (Dallolio, Ceciliani, Sanna, Garulli y Leoni, 2016). Este resultado podría explicarse por el mayor trabajo planificado en este programa para los miembros inferiores con respecto a los superiores y por la duración de la intervención, que parece ser un factor determinante en el efecto de este tipo de programas sobre la composición corporal puesto que sólo programas de larga duración parecen incidir en la disminución de la masa grasa (Bicanin, et al, 2018).

El programa se diseñó con el objetivo de mejorar dos aspectos fundamentales para contribuir a que los niños sean activos: la mejora de la autoestima y la intención de seguir siendo activo. Las clases de EF deben crear un contexto en el que los alumnos experimenten un contacto con la AF orientado a ser activos a medida que comprueban los beneficios de la práctica. Las actitudes positivas que desarrollen dentro del contexto escolar influirán en la generación o no de hábitos de práctica perdurables (Deem y Gilroy, 1998). Estos objetivos se tuvieron en cuenta en el planteamiento de la intervención a través del diseño de las actividades y la metodología de enseñanza. Sin embargo, a pesar de que el programa mostró mejoras en los aspectos valorados en el cuestionario de intención de seguir siendo activo, éstas no fueron significativas. Una posible explicación puede encontrarse en el alto índice de práctica de AF del grupo experimental, muy superior a la media en escolares españoles, así como a la elevada puntuación de partida (Trudeau y Shephard, 2005).

En cuanto a la autoestima, valorada a partir de las puntuaciones de tres de sus dimensiones (socioemocional, académico y deportiva), el grupo experimental mostró mejoras después del programa en todas ellas, aunque sólo fueron estadísticamente significativas en el caso de la socioemocional. En contrapartida, en el grupo control se halló descenso de todas en el potstest. Estos resultados evidencian el esfuerzo en el diseño de las actividades priorizando la interacción del alumnado y el trabajo cooperativo. Por otra parte, son similares a los encontrados por Contreras et al. (2014) quienes no observaron diferencias en el autoconcepto físico después de la intervención, y parcialmente compatibles con los hallados por Kyle et al. (2016), quienes encontraron ligeras mejoras en el autoconcepto físico y el académico, aunque tras un programa de 7 meses de duración. Por otra parte, analizando los resultados del grupo control, donde se destaca un descenso de la autoestima, característico del inicio de la adolescencia, el efecto del programa sobre la autoestima en el grupo experimental tendría un valor ańadido a pesar de no ser estadísticamente significativo en alguna de sus dimensiones. 
Como conclusión destacamos que en el contexto escolar un programa intencional de AF puede contribuir a favorecer la CF de los escolares, la autoestima e incluso la intención de ser activo, con los beneficios físicos, psicológicos y sociales que puede tener para su salud.

Algunas limitaciones de este estudio fueron la duración de la intervención y las características del grupo experimental. Programas de intervención más duraderos parecen ser más efectivos, además, los participantes mostraron índices de AF y de intención de seguir siendo activos muy elevados, pudiendo condicionar los resultados obtenidos.

\section{Referencias}

1. Abad, B., y Cañada, D. (2014). Unidades Didácticas Activas. Ministerio de sanidad, servicios sociales e igualdad. Centro de publicaciones.

2. Álvarez-Bueno, C., Pesce, C., Cavero-Redondo, I., Sánchez-López, M., Garrido-Miguel, M. y Martínez-Vizcaíno, V., (2017). Academic Achievement and Physical Activity: A Meta-analysis. Pediatrics, 140(6), e20171498.

3. Bicanin, P., Milenkovic, S., Radovanovic, D., Gajevic, A. y Ivanovic, J. (2018). Effects of programmed firness exercise on body composition among pre-school children. Physical Education and Sport, 16(1), 47-56.

4. Camacho-Miñano, M.J., Fernández García, E., Ramírez, E., y Blández, J. (2013). La Educación Física escolar en la promoción de la actividad física orientada a la salud en la adolescencia: una revisión sistemática de programas de intervención. Revista Complutense de Educación, 24(1), 9-26.

5. Contreras, O.R., Pastor-Vicedo, J.C., Gil, P. y Tortosa, M. (2014). Intervención escolar para corregir el sobrepeso y la obesidad. Diseño, implementación y evaluación de un programa de educación física para primer ciclo de ESO. Trauma 25(4), 200-204.

6. Currie, C., Gabhainn, S. N. y Godeau, E. (2008). Inequalities in young people's health. Health behaviour in school-aged children. (pp. 105112). Copenhagen, Denmark: WHO Regional Office for Europe.

7. Dallolio, L., Ceciliani, A., Sanna, T., Garulli, A. y Leoni, E. (2016). Proposal for an Enhanced Physical Education Program in the Primary School: Evaluation of Feasibility and Effectiveness in Improving Physical Skills and Fitness. Journal of Physical Activity and Health, 13,10251034.

8. Deem, R. y Gilroy, S. (1998). Physical Activity, Life-Long Learning and Empowerment: Situating Sport in Women's Leisure. Sport, Education and Society, 3, 89-104

9. Diamond, A.B. (2015). The cognitive benefits of exercise in youth. American College of Sport Medicine, 14(4), 320-6.

10. Eather, N., Morgan, P.J. y Revalds, D. (2016). Improving health-related fitness in adolescents: the CrossFit TeensTM randomised controlled trial. Journal of Sports Sciences, 34(3), 209-223.

11. Estero, J. L. A., Oliva, F. J. C., y Lucas, J. L. Y. (2013). Propiedades psicométricas de la escala de intencionalidad de ser físicamente activo en Educación Primaria. Revista de Educación, 362, 485-505.

12. Faison-Hodge, J. y Porretta, D.L. (2004). Physical activity levels of students with mental retardation and students without disabilities. Adapted Physical Activity Quarterly, 21, 139-154.

13. Fernández-Bustos, J.G., y Abellán, J. (2016). Los juegos y deportes alternativos. En Gil, P. y Abellán, J. (Eds). Mediación Educativa. Juegos, Ocio y Recreación (pp 115-128). Madrid: Pirámide. I.S.B.N.: 978-84368-3610-3

14. Fernández-Bustos, J. G., Infantes-Paniagua, Á., Cuevas, R., y Contreras, O. R. (2019). Effect of Physical Activity on Self-Concept: Theoretical Model on the Mediation of Body Image and Physical SelfConcept in Adolescents. Frontiers in Psychology, 10, 1537. doi: 10.3389/ fpsyg.2019.01537

15. García-Cantó, E. y García-López, A. (2010). El autoconcepto y la práctica de actividad física en primaria. Trances, 3(2), 187-200.

16. Guillamón, A.R., Garcia-Cantó, E. y Carrillo, P.J. (2019). Actividad física, condición física y autoconcepto en escolares de 8 a 12 años. Retos, 35, 236-241.

17. Guillamón, A.R., García-Cantó, E., Pérez, J.J. y Rodríguez, P.L. (2015). Capacidad aeróbica y su relación con parámetros de la condición física saludable en escolares. Revista Facultad de Ciencias de la Salud UDES, 2(2), 90-96.

18. Hein, V., Müür, M., y Koka, A. (2004). Intention to be physically active after school graduation and its relationship to three types of intrinsic motivation. European Physical Education Review, 10, 5-19.

19. Janssen, I. y LeBlanc, A.G. (2010). Systematic review of the health benefits of physical activity and fitness in school-aged children and youth. International Journal of Behavioral Nutrition and Physical Activity, 7(40), 1-16.

20. Janz, K.F., Dawson, J.D. y Mahoney, L.T. (2002). Increases in Physical Fitness During Childhood Improve Cardiovascular Health During Adolescence: the Muscatine Study. International Journal of Sports Medicine, 23(S1), 15-21.

21. Kyle, T.L., Hernández, A., Reigal, R.E., Morales, V. (2016). Efectos de la actividad física en el autoconcepto y la autoeficacia en preadolescentes. Retos, 29, 61-65.

22. Londsdale, C., Rosenkranz, R.R., Peralta, R.L., Bennie, A., Fahey, P. y Lubans, D.R. (2013). A systematic review and meta-analysis of interventions designed to increase moderate-to-vigorous physical activity in school physical education lessons. Preventive Medicine, 56, 152-161.

23. Manchola-González, J., Bagur-Calafat, C., y Girabent-Farrés, M. (2017). Fiabilidad de la versión española del cuestionario de actividad física PAQ-C. Revista Internacional de Medicina y Ciencias de la Actividad Fisica y el Deporte 17(65), 139-152

24. Mocha, J.A., Garcés, S.E., Medina, S.V., Castro, W.E. y Suntasig, M.G. (2018). Análisis del consumo máximo de oxígeno (vo2máx) post intervención de un programa en juegos pre-deportivos. Ciencia Digital, 2(2), 232-247.

25. Organización de las Naciones Unidas para la Educación, la Ciencia y la Cultura, UNESCO, Educación Física de Calidad (EFC): guía para los responsables políticos (París, 2015), https:/unesdoc.unesco.org/ ark:/48223/pf0000231340

26. Ortega, F.B., Artero, E.G., Ruiz, J.R., Espańa-Romero, V., JiménezPavón, D., Vicente-Rodriguez, G., ... Castillo, M.J. (2011). Physical fitness levels among European adolescents: the HELENA study. British Journal of Sports Medicine, 45, 20-29.

27. Ortega, F.B., Ruiz, J.R., Castillo, M.J., y Sjöström, M. (2008). Physical fitness in childhood and adolescence: a powerful marker of health. International Journal of Obesity, 32, 1-11

28. Powell, E., Woodfield, L.A. y Nevill, A.M. (2016). Increasing physical activity levels in primary school physical education: The SHARP Principles Model. Preventive Medicine Reports, 3, 7-13.

29. Ruiz, J.R., Espańa, V., Castro, J., Artero, E.G., Ortega, F.B., Cuenca, M., ...Castillo, M.J. (2011). Batería ALPHA-Fitness: test de campo para la evaluación de la condición física relacionada con la salud en niños y adolescentes. Nutrición hospitalaria, 26(6), 1210-1214.

30. Shavelson, R.J., Hubner, J.J., y Stanton, G.C. (1976). Self-Concept: Validation of Construct Interpretations. Review of Educational Research, 
46(3), 407-441.

31. Siedentop, D. (1994). Sport education: Quality PE through positive sport experiences. Champaign, IL: Human Kinetics.

32. Strong, W.B., Malina, R.M., Blimkie, C.J., Daniels, S.R., Dishman, R.K., Gutin, B., ... Trudeau, F. (2005). Evidence based physical activity for school-age youth. The Journal of Pediatrics, 146(6), 732-7.

33. Trudeau, F. y Shephard, R.J. (2005). Contribution of school programmes to physical activity levels and attitudes in children and adults. Sports Medicine, 35(2), 89-105. 